\title{
A Mathematical Model ForProlactin Levels In Olanzapine Treatment Of Schizophrenia Patients
}

\author{
${ }^{1,}$ S.Lakshmi , and ${ }^{2, M}$.Goperundevi \\ ${ }^{1,}$ Principal and Research Advisor, Govt. Arts and Science College,Peravurani - 614 804, \\ Thanjavur- District, Tamilnadu. \\ ${ }^{2,}$ Research Scholar, P.G \& Research Dept. of Mathematics, K.N.Govt. Arts College for \\ Women (Autonomous), Thanjavur-613 007.
}

\begin{abstract}
Elevated prolactin levels may play important roles, both direct and indirect in various pathologic states, including breast cancer, oesteoporosis, cardiovascular disorders, sexual disturbances and schizophrenia. In our previous paper[7]a two-step strategy for modeling degradation for elevated prolactin levels in patients with Schizophrenia with an initiation period is proposed. As a result we can identify which location parameter needs to be increased in order to improve the overall reliability. Here, in the application part the study was designed to investigate the relationship between the treatment effect of olanzapine and the serum prolactin level in schizophrenia and to investigate the factors that may act as predictors of response for olanzapine treatment. As a result the curves for the availability function and the failure rate density function are obtained for the corresponding medical curve given in the application part.
\end{abstract}

KEYWORDS: Schizophrenia, Olanzapine, Prolactin, Antipsychotics, Availability Functions.

Mathematical Subject Classification: $\quad 60 \mathbf{G}_{\mathrm{xx}}$

\section{MATHEMATICAL MODEL}

Assumptions:

1. The time till failure has an exponential distribution $E(\beta)$

2. The time till repair has an exponential distribution $E(\gamma)$. Let $\lambda=\frac{1}{\beta}$ and $\mu=\frac{1}{\gamma}$. Accordingly,

And

$$
f(t)= \begin{cases}0, & t \leq 0 \\ \lambda e^{-\lambda t}, & t>0\end{cases}
$$

$$
g(t)= \begin{cases}0, & t \leq 0 \\ \mu e^{-\mu t}, & t>0\end{cases}
$$

The corresponding Laplace transforms are $f^{*}(s)=\lambda /(\lambda+s)$ and $g^{*}(s)=\mu /(\mu+s)$, respectively. According to the equation

$$
v^{*}(s)=\frac{f^{*}(s) g^{*}(s)}{1-f^{*}(s) g^{*}(s)} 0<s<\infty .
$$

the Laplace transform of the renewal density is

$$
v^{*}(s)=\frac{\lambda \mu}{s^{2}+(\lambda+\mu) s}=\frac{\lambda \mu}{(\lambda+\mu)}\left(\frac{1}{s}-\frac{1}{s+\lambda+\mu}\right) \ldots
$$

Every Laplace transform $f^{*}(s)$ on $(0, \infty)$ has a unique inverse $\mathrm{f}(\mathrm{t})$ on $(0, \infty)$.

The inverse transform of (1.2) can be easily checked as

$$
v(t)=\frac{\lambda \mu}{(\lambda+\mu)}-\frac{\lambda \mu}{(\lambda+\mu)} e^{-t(\lambda+\mu)}, 0<t<\infty .
$$

In a similar fashion we obtain the failure density

$$
w(t)=\frac{\lambda \mu}{(\lambda+\mu)}+\frac{\lambda^{2}}{(\lambda+\mu)} e^{-t(\lambda+\mu)}, 0<t<\infty
$$

Integrating (2.2) and (2.3) we obtain, for $0<t<\infty$

$$
\begin{aligned}
& V(t)=\frac{\lambda \mu}{(\lambda+\mu)} t-\frac{\lambda \mu}{(\lambda+\mu)^{2}}\left(1-e^{-t(\lambda+\mu)}\right), \\
& \text { And } \\
& W(t)=\frac{\lambda \mu}{(\lambda+\mu)} t+\frac{\lambda^{2}}{(\lambda+\mu)^{2}}\left(1-e^{-t(\lambda+\mu)}\right),
\end{aligned}
$$


Finally the unavailability function is $Q(t)=W(t)-V(t)$

$Q(t)=\frac{\lambda}{(\lambda+\mu)}-\frac{\lambda}{(\lambda+\mu)} e^{-t(\lambda+\mu)}, 0<t<\infty$.

And the availability function is $A(t)=1-Q(t)$

$$
A(t)=\frac{\mu}{(\lambda+\mu)}+\frac{\lambda}{(\lambda+\mu)}\left(e^{-t(\lambda+\mu)}\right), 0<t<\infty \ldots
$$

Notice that $\lim _{t \rightarrow \infty} A(t)=A_{\infty}=\frac{\mu}{\lambda+\mu}=\frac{\beta}{\beta+\gamma}$

Generally the availability function $\mathrm{A}(\mathrm{t})$ is given by the formula

$$
A(t)=1-F(t)+\int_{0}^{t} v(x)[1-F(t-x)] d x
$$

$\operatorname{Or} A(t)=R(t)+\int_{0}^{t} v(x) R(t-x) d x$

Where $\mathrm{F}(\mathrm{t})$ is the CDF of the TTF and $\mathrm{R}(\mathrm{t})$ is the reliability function of a non repairable system[2]. Thus, if $R^{*}(s)$ is the Laplace transform of the reliability function and $A^{*}(s)$ is that of availability function, we obtain from (1.1) and (1.10) that

$$
\begin{gathered}
A^{*}(s)=R^{*}(s)\left(1+v^{*}(s)\right) \\
A^{*}(s)=\frac{R^{*}(s)}{1-f^{*}(s) g^{*}(s)}, 0<s<\infty \ldots \ldots \ldots \ldots
\end{gathered}
$$

The Laplace transform of (2.11) should be inverted either analytically or numerically to obtain the availability function of the system. We derived the availability function of a system whose TTF is exponentially distributed, $\mathrm{E}(\beta)$, and the TTR is exponentially distributed $\mathrm{E}(\gamma)$. The availability function of this system is given in (1.8). In this model we derive the availability function of a system whose TTF has Erlang distribution G(2, $\beta$ ), and whose TTR is exponentially distributed $\mathrm{E}(\gamma)$. This model can represent a system with a standby unit which is switched on automatically upon the failure of the functional unit Repair, however, is delayed until both units fail, which is a failure time for the whole system[1]. The system is renewed when both units are repaired. Let $\lambda=1 / \beta$ and $\mu=1 / \gamma$. We assume that $\lambda<\mu / 4$. Hence the reliability of the system is $R(t)=e^{-\lambda t}+$ $\lambda t e^{-\lambda t}, 0<t<\infty$. The Laplace transform of this reliability function is

The Laplace transform of the PDF of the TTF is

$$
R^{*}(s)=\frac{2 \lambda+s}{(\lambda+s)^{2}}
$$

$$
f^{*}(s)=\left(\frac{\lambda}{\lambda+s}\right)^{2}
$$

And that of the TTR is $g^{*}(s)=\frac{\mu}{\mu+s}$. Thus, according to (1.11), the Laplace transform [8] of the availability function is

$$
\begin{array}{r}
A^{*}(s)=\frac{s^{2}+(2 \lambda+\mu) s+2 \lambda \mu}{s\left[s^{2}+(2 \lambda+\mu) s+\left(\lambda^{2}+2 \lambda \mu\right)\right]} \ldots \\
=\frac{p(s)}{s q(s)}, \quad 0<s<\infty
\end{array}
$$

Notice that $q(s)=p(s)+\lambda^{2}$. Let $\mathrm{s}_{1}$ and $\mathrm{s}_{2}$ be the two roots of the second order polynomial $\mathrm{q}(\mathrm{s})$. These roots are

$$
\begin{aligned}
& s_{1}=-\left(\lambda+\frac{\mu}{2}\right)+\frac{1}{2}(\mu(\mu-4 \lambda))^{1 / 2} \\
& s_{2}=-\left(\lambda+\frac{\mu}{2}\right)-\frac{1}{2}(\mu(\mu-4 \lambda))^{1 / 2}
\end{aligned}
$$

From the tables of Laplace transforms the inverse of $A^{*}(s) i s A(t)$ and $\mathrm{A}(\mathrm{t})$ is given as below,

$$
A(t)=\frac{2 \lambda \mu}{\lambda^{2}+2 \lambda \mu}-\frac{\lambda^{2}}{s_{1}\left(s_{1}-s_{2}\right)} e^{s_{1} t}-\frac{\lambda^{2}}{s_{2}\left(s_{2}-s_{1}\right)} e^{s_{2} t}, 0<t<\infty .
$$

Notice from the equations (1.15) \& (1.16) both the roots $s_{1}$ and $s_{2}$ are negative. Thus, the asymptotic availability of the system under consideration is $A_{\infty}=\frac{\mu}{\mu+\lambda / 2}$.

From equations (1.6) \& (1.17) we can find out the mathematical representation of the Availability Function and the Failure Rate Density. 


\section{APPLICATION}

Prolactin is an anterior pituitary peptide hormone that has a major role in preparing the breast for lactation and maintenance of lactation. This hormone, thus, has great physiologic importance during pregnancy and breast feeding[3]. The synthesis and release of prolactin in inhibited by hypothalamic dopamine, which acts which acts on $\mathrm{D}_{2}$ receptors located on the surface of the lactotroph cells. Any process that results in a reduction in the dopamine influence of the lactotroph cell $\mathrm{D}_{2}$ receptors would increase prolactin levels [6].The relative risk of an elevated prolactin level needs to be considered in the treatment decision process. All antipsychotic drugs may affect serum prolactin levels to some degree. Prolactin - elevating antipsychotic drugs generally include all typical antipsychotics and risperidone. Prolactin - sparing antipsychotic drugs include clozapine, olanzapine, and quetiapine. The serum prolactin level may be used as an indicator of a blockade of $\mathrm{D}_{2}$ receptors, and thus as a measure of the bioavailability of typical and atypical antipsychotics. Monoamine Oxidase (MAO) is a mitochondrial enzyme involved in the degradation of biological amines, including dopamine, serotonin, and norepinephrine[4]. A recent study supports the involvement of the MAOA gene with schizophrenia[9].

Based on these studies, the principal goal of this study is to test the following hypothesis:

[1] The serum level of prolactin and the clinical improvement in Positive And Negative Syndrome Scale (PANSS)[5] Score are related.

[2] We also considered that the genetic factor would be related to the serum prolactin.

This study used a monotherapy design in a homogenous group of patients, and applied the Generalized Estimating Equations - I (GEE - I)[11]. The primary objective of this analysis was to explore the relation between clinical effects and prolactin level and to investigate the factors that may act as predictors of response for olanzapine treatment.

\section{Methodology}

Sixty patients (36 men, 24 women) who met the diagnostic criteria for schizophrenia according to the Diagnostic and Statistical Manual of Mental Disorders were assigned to treatment with olanzapine in a 3 month study. Patients with schizophrenia who had discontinued their previous oral antipsychotics on their own for a period of at least 7 days were recruited as possible subjects.

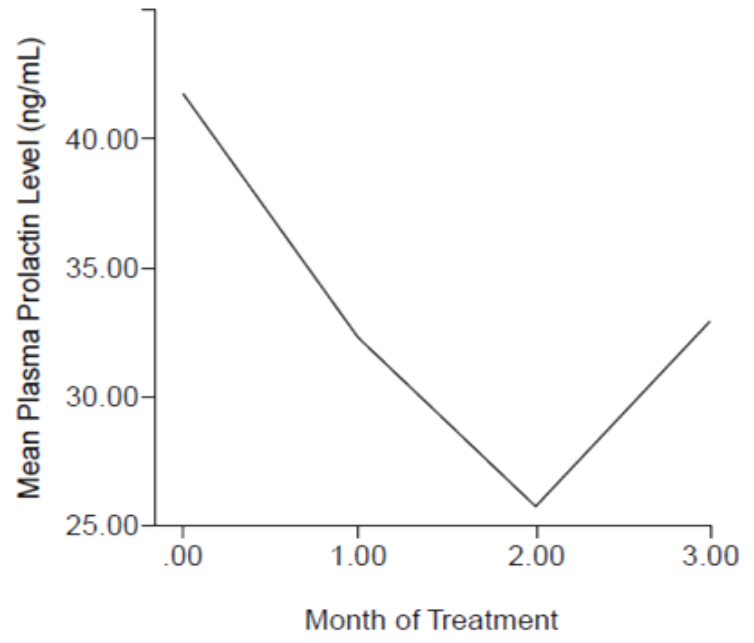

Fig. 2.1.1

\section{Mean Prolactin level during each month of study in schizophrenia patients with olanzapine treatment.}

A monotherapy (olanzapine) study design was used with a homogenous group of patients and was started with a run - in period of 3 months. Patients were required to have discontinued using either typical or atypical antipsychotics for at least 7 days prior to the study entry. The patients received olanzapine treatment over a period of 3 months. The dose of olanzapine was flexible, ranging from 10 to $30 \mathrm{mg}$ p.o. daily. Serum prolactin levels were assessed at baseline (after drug washout) and at months 1,2 and 3 during olanzapine treatment.Fasting morning blood specimens for the assays of prolactin were drawn at baseline and every month thereafter (at the end of month 1, month 2 and month 3), consecutively. The baseline data were measured immediately before starting monotherapy treatment (day 0 ). In addition, the clinical effect was evaluated by assessing the patient's symptoms at baseline (following drug washout), and every month thereafter 3 months, using the PANSS. 
The PANSS includes 30 items that measure positive symptoms, negative symptoms, and general psychopathology. Supplementary items for the aggression risk profile included anger, difficulty in delaying gratification, and affective liability.

\section{RESULTS:}

As indicated in Figure 2.1.1, olanzapine treatment was associated with a decrease in the serum prolactin level in the first 2 months and a mild increase at the end of the third month. But in general, the prolactin level during the third month was relatively lower than the baseline level. The mean (SD) baseline serum prolactin level was $41.63(23.09) \mathrm{ng} / \mathrm{ml}$, the first month was $32.20(18.02) \mathrm{ng} / \mathrm{ml}$, the second month was $25.72(16.77) \mathrm{ng} / \mathrm{ml}$ and the third month was $32.86(20.48) \mathrm{ng} / \mathrm{ml}$. We demonstrated that there was no significantly close correlation between serum prolactin level and any of the PANSS items and the PANSS subscore (general, positive, excitement, and aggression), except the positive subscore. There was a close correlation between the serum prolactin level and clinical improvement in positive symptoms: the more decreased the serum prolactin level, the more improvement in positive symptoms $(\mathrm{p}=0.002)$. In the present study it is demonstrated that there is a positive correlation between the serum prolactin level and the positive symptoms in female schizophrenia patients. It is also found as that serum prolactin has no significant association with the MAOA polymorphism and DRD4 genotype. The measurement of the GEE - I model and the working correlation matrix in this 3 - month of trial of olanzapine ranged from 0.139 to 0.519 .

\section{Medical Findings}

The main findings of this study were:

[1] Olanzapine treatment significantly decreased the serum prolactin level,

[2] The prolactin level showed a more pronounced decrease in olanzapine- treated male schizophrenia patients than in female patients, and

[3] There was a close relationship between the improvement in positive symptoms and the change in serum prolactin level before and after olanzapine treatment.

The results of this study suggest that olanzapine was effective in reducing the severity of overall psychotic symptoms and that olanzapine treatment significantly decreased prolactin levels in schizophrenia patients. The data indicate that in a 3 - month olanzapine treatment period, a flexible dose (ranging from 10 to 30 $\mathrm{mg} /$ p.o.) causes a decrease in prolactin levels in schizophrenia patients. On the basis of the result, olanzapine seems to be superior to typical and some prolactin elevating atypical antipsychotics in terms of this endocrinologic side effect. The findingindicates that female schizophrenia patients, when treated with olanzapine, show significantly more prolactin secretion than male schizophrenia patients. These results may be due toelevated prolactin values at lower reference doses in female than in male patients [10]. In this study it is found that these genetic factors have no association with the plasma prolactin level. This may be the result of olanzapine treatment. When evaluating the serum prolactin level change in these olanzapine - treated patients, it is most important to understand that the baseline levels of prolactin may have been affected by various pre-study antipsychotics. This study in chronic schizophrenia demonstrated that there was a close relationship between the improvement in positive symptoms in female patients and the change in the serum prolactin level before and after olanzapine treatment. Because the level of serum prolactin tended to decrease the more positive symptoms improved, drug - induced prolactin secretion should be considered when treating schizophrenia patients with antipsychotics, and prolactin - elevating drugs should be avoided. Recognizing antipsychotic drugs with a favorable prolactin profile would be important in alleviating the morbidities associated with drug - induced prolactin elevation.

\section{MATHEMATICAL RESULTS:}




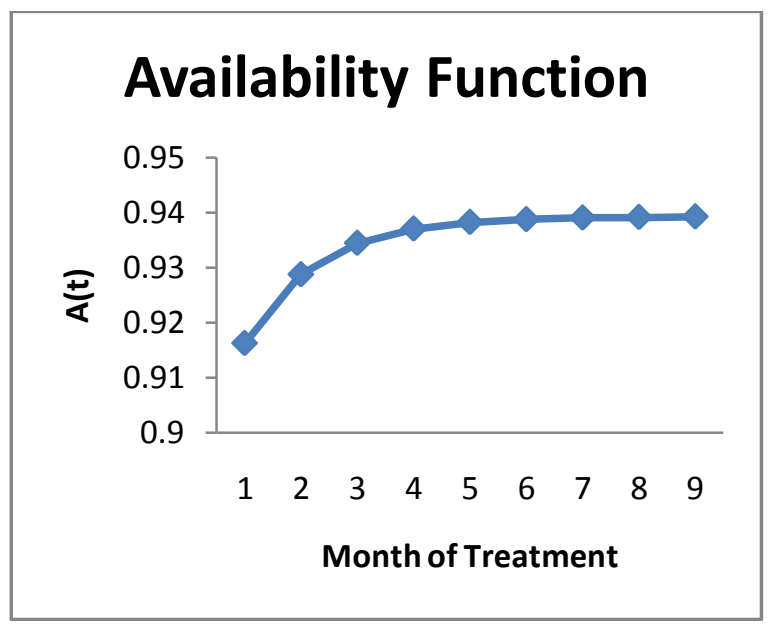

\section{Failure Rate Density}

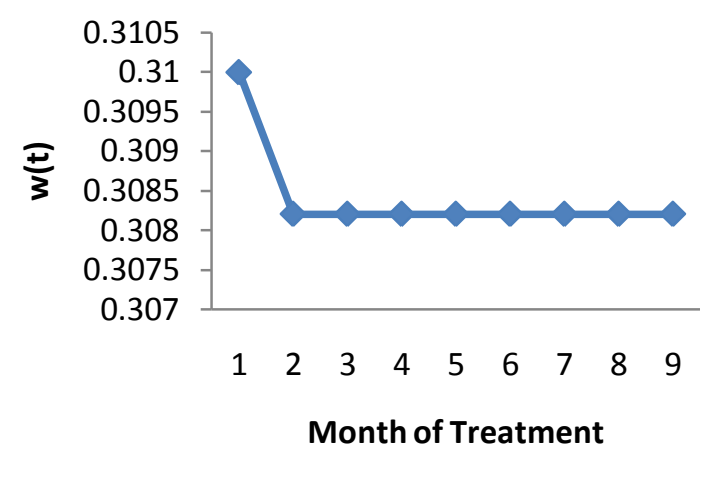

Fig. 3.1

Fig. 3.2

From the above figures it is known that $\mathrm{A}(\mathrm{t})$ increases as $\mathrm{t}$ increases from the first month onwards and $\mathrm{W}(\mathrm{t})$ attains its maximum value at the end of the first month and get a sudden decrease and it becomes stable from the second month till the end of the treatment.

\section{CONCLUSION}

As a result the curves for the availability function and the failure rate density are obtained in Figure 3.1 \& Figure 3.2 for the corresponding medical representation which was given in the application part. During the treatment period (in months) the availability function increases monotonically from the end of the first month and it increases gradually till the end of the treatment, and the failure rate density undergo a sudden fall in the first month of treatment and it is stable till the end of the treatment. When evaluating the serum prolactin level change in these olanzapine - treated patients, it is most important to understand that the baseline levels of prolactin may have been affected by various pre-study antipsychotics. This study in chronic schizophrenia demonstrated that there was a close relationship between the improvement in positive symptoms in female patients and the change in the serum prolactin level before and after olanzapine treatment. Because the level of serum prolactin tended to decrease the more positive symptoms improved, drug - induced prolactin secretion should be considered when treating schizophrenia patients with antipsychotics, and prolactin - elevating drugs should be avoided.

\section{REFERENCES}

[1] Ascher H, Feingold H, "Repairable systems reliability: Modeling inference, New York, Marcel Dekker.

[2] Crow L.H, "Evaluating the reliability of repairable systems", In Proceedings of the Annual Reliability and Maintainability Symposium: 1990 (pp: 275 - 279)

[3] Dickson R.A, Glazer W.M, Neuroleptic - induced hyperprolactinemia. Schizophr Res 1999; 35: S75 - S86

[4] Jonson E.G, Norton N, Forsland K, et al., Association between a promoter variant in the monoamine Oxidase A gene and schizophrenia. Schizophr Res. 2003; 61(1): $\quad 31-37$.

[5] Kay S.R, Opler L.A, Fiszbein A. Significance of positive and negative syndromes in chronic schizophrenia. Br J Psychiatry 1986; 149: 439 - 448.

[6] Kinon B.J, Gilmore J.A, Liu H, et al., Hyperprolactinemia in response to antipsychotic drugs: characterization across comparative clinical trials. Psychoneuroendocrinology 2003; 28: $69-82$.

[7] Lakshmi S, Goperundevi M, "A Mathematical Degradation Model for Elevated Prolactin Levels in Patients with Schizophrenia", American Journal of Mathematics and Mathematical Sciences, Volume - 2, Number - 2, July - December 2013, Pp: 107 - 113.

[8] Lewis E.E, "Introduction to Reliability Engineering”, New York, Wiley, 1987.

[9] Lung F.W, Tzeng D.S, Shu B.C, Ethnic heterogeneity in allele variation in the DRD4 Schizophr Res. 2005; 57: 239 - 245.

[10] Melkersson K.I, Hulting A.L, Rane A.J, Dose requirement and prolactin elevation on antipsychotics in male and female patients with schizophrenia or related psychoses. Br J ClinPharmacol 2001; 51: 317 - 324

[11] Zeger S.L, Liang K.Y, Longitudinal and data analysis for discrete and continuous outcomes, Biometrics 1986; 42(1): $121-130$. 